\title{
Inspection of Cylindrical Structures Using the First Longitudinal Guided Wave Mode in Isolation for Higher Flaw Sensitivity
}

\author{
Premesh Shehan Lowe, Student Member, IEEE, Ruth M. Sanderson, \\ Nikolaos V. Boulgouris, Senior Member, IEEE, Alex G. Haig, \\ and Wamadeva Balachandran, Fellow, IEEE
}

\begin{abstract}
Less attention has been given to the inspection using the first longitudinal guided wave mode due to its attenuative and dispersive properties at commonly used ultrasonic guided waves (UGWs) operating frequency region $(20-100 \mathrm{kHz}$ ). However, the first longitudinal guided wave mode has higher flaw sensitivity due to having a shorter wave length and having higher number of non-axisymmetric wave modes at a given frequency. This enhances the capabilities of advanced UGW techniques which require higher number of non-axisymmetric modes. This study has been performed to investigate the potential of mode purity and flaw sensitivity of the first longitudinal guided wave mode compared with other axisymmetric modes in the UGW operating frequency region. Numerical and experimental investigation have been conducted to investigate pure excitation and flaw sensitivity of the first longitudinal guided wave mode. It has been validated that the first longitudinal guided wave mode can be used in the UGW inspection effectively in isolation by adopting transducers with out-of-plane vibration. This reduces the cost and the weight of the UGW inspection tooling. The flaw sensitivity of the first longitudinal guided wave mode has been investigated by aid of an empirically validated UGW focusing technique. Under the studied conditions in this paper, the first longitudinal guided wave mode has $\sim 5$ times higher flaw sensitivity compared with the second longitudinal guided wave mode and $\sim 2.5$ times higher than the first torsional guided wave mode. This enhances the capability of UGW flaw detection and sizing.
\end{abstract}

Index Terms-Pipeline inspection, ultrasonic guided waves, compression transducers, first longitudinal guided wave mode, ultrasonic guided wave focusing.

\section{INTRODUCTION}

$\mathbf{R}$ ESEARCH on UGW inspection has expanded over recent decades including the use of low frequency ultrasound to screen large specimens e.g. pipes. Pipelines are

Manuscript received June 15, 2015; revised September 30, 2015; accepted October 1, 2015. Date of publication October 14, 2015; date of current version January 12, 2016. This work was supported in part by TWI Ltd., and in part by the Center for Electronic System Research, Brunel University. The associate editor coordinating the review of this paper and approving it for publication was Dr. Stefan J. Rupitsch.

P. S. Lowe is with Brunel University, Middlesex UB8 3PH, U.K., and also with TWI Ltd., Cambridge CB21 6AL, U.K. (e-mail: eepgpsw@brunel.ac.uk).

R. M. Sanderson and A. G. Haig are with TWI Ltd., Cambridge CB21 6AL, U.K. (e-mail: ruth.sanderson@twi.co.uk; alex.haig@twi.co.uk).

N. V. Boulgouris and W. Balachandran are with Brunel University, Middlesex UB8 3PH, U.K. (e-mail: nikolaos.boulgouris@brunel.ac.uk; wamadeva.balachandran@brunel.ac.uk).

Digital Object Identifier 10.1109/JSEN.2015.2487602 widely used to transport energy products such as natural gas and crude oil. As pipelines age, corrosion flaws can develop and it is therefore important to find techniques to inspect them efficiently. Pipelines in the industries are commonly inaccessible and insulated. Because of that, UGW inspection has become more attractive as a non-destructive testing technique in the past two decades. Guided wave based techniques offer the advantage of full volume inspection from a single test location. However, there is a clear need to achieve higher flaw sensitivity to enhance the inspection resolution.

The UGW technique works on elongated geometries with a constant cross-section, e.g. pipes. The sound generated in these geometries is constrained to propagate along the axis of the body, and similarly return back to the generation position when an impedance change (discontinuity) is encountered. UGW exist because there is a medium between boundaries for the wave to propagate. These boundaries are said to form a 'waveguide'. Depending on the waveguide geometry, material properties and the excitation frequency, the possible number of wave modes will vary. UGW can be used to inspect engineering structures e.g. plates, rods, rails and pipes and this study is focused on UGW inspection of pipelines.

Nomenclatures for identifying guided wave modes in cylindrical structures are essential as there are a high number of wave modes with varying displacement characteristics. The nomenclature used throughout this study was suggested by Meitzler [1] and popularized by Silk and Bainton [2]. According to this nomenclature, vibration modes in cylindrical structures are based on the following format, $X(n, m)$. $X$ represents the character to denote whether the vibration modes are longitudinal and axisymmetric $(L)$, torsional and axisymmetric $(T)$, or non-axisymmetric (flexural) $(F)$. The $n$ is a positive integer giving the identification of harmonic variations of displacement around the circumference and $m$, again a positive integer, is to indicate the incremental order of the modes of vibration within the wall. In typical pipeline sizes and test frequencies $(20-100 \mathrm{kHz})$ the three axisymmetric guided wave modes that can be excited are $\mathrm{L}(0,1), \mathrm{L}(0,2)$ and $\mathrm{T}(0,1)$ [3]. Longitudinal axisymmetric wave modes are denoted by $\mathrm{L}(0, m)$ and torsional axisymmetric wave modes are denoted by $\mathrm{T}(0, m)$. Non-axisymmetric flexural wave modes are denoted by $\mathrm{F}(n, m)$. Furthermore, the torsional wave mode has circumferential displacement, whereas the longitudinal 


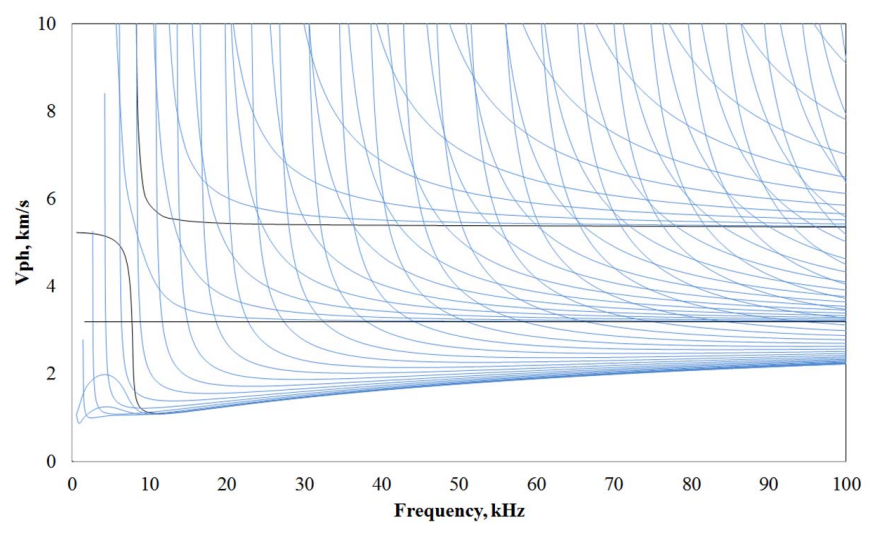

Fig. 1. Phase velocity dispersion curves for a nominal 8 inch schedule 40 (outer diameter: $219.1 \mathrm{~mm}$ and wall thickness: $8.18 \mathrm{~mm}$ ) steel pipe.

wave modes have axial and radial displacement (generally the $\mathrm{L}(0,2)$ has predominantly axial displacement and the $\mathrm{L}(0,1)$ has both axial and radial displacement) [2], [3].

The resolution of the UGW inspection is affected by the dispersion and the attenuation of the wave modes. Dispersion of UGW can occur as they propagate through the test structure. Dispersion can limit inspection resolution because of losses in signal amplitude relative to the noise level. Also, the level of dispersion in a particular wave mode will affect the data interpretation as the reflection can become distorted [4], [5]. The so-called dispersion curve graphs illustrate the wave velocity in relation to the frequency (or wavenumber) with separate curves for each of the existing wave modes in a frequency region. With regards to pipe wave modes, each of the axisymmetric modes has a family of flexural wave modes. Fig. 1 illustrates the phase velocity dispersion curves for a nominal 8 inch schedule 40 steel pipe (outer diameter: $219.1 \mathrm{~mm}$ and wall thickness: $8.18 \mathrm{~mm}$ ) calculated using the RAPID (Rapid Automated Pipe Dispersion Curve Generator) software [6]. In Fig. 1, black lines represent the axisymmetric modes present in the UGW typical operating frequency region $(20-100 \mathrm{kHz})$ and blue lines represents family of flexural wave modes. As shown in Fig. 1, the $\mathrm{T}(0,1)$ wave mode is nondispersive at all frequencies and the $\mathrm{L}(0,2)$ wave mode is non-dispersive at some frequency regions $(20 \mathrm{kHz}-100 \mathrm{kHz}$ in this example). Because of that, $\mathrm{T}(0,1)$ and $\mathrm{L}(0,2)$ are the modes that are most commonly used UGW wave modes for inspection. The $\mathrm{L}(0,1)$ mode can be dispersive [7] and attenuative [8] in the frequency regions commonly used for UGW inspection. For this reason the $\mathrm{L}(0,1)$ wave mode has gained less attention for UGW inspection in favour of $\mathrm{T}(0,1)$ and $\mathrm{L}(0,2)$.

Early research into the use of first longitudinal UGW mode in cylindrical structures has been performed by Silk and Bainton [2]. They studied cylindrical guided wave modes [9], [10] and reported their equivalence to lamb waves. They studied the behaviour of the $\mathrm{L}(0,1)$ propagation and also the $\mathrm{L}(0,1)$ propagation around a bend ( $\mathrm{U}-$ form). Attenuation of $\mathrm{L}(0,1)$ has been studied by $\mathrm{Na}$ and Yoon [8]. They studied the attenuation effect with different fluids in the pipe and presented the attenuation curves based on the numerical calculations.
They also observed that the attenuation is considerably low in the frequency region of $20-100 \mathrm{kHz}$. Dispersion of the first two longitudinal modes, $\mathrm{L}(0,1)$ and $\mathrm{L}(0,2)$ wave modes were studied empirically in a cylindrical structure filled with liquid [7]. They observed that the dispersion curves of the $\mathrm{L}(0,2)$ wave mode dramatically alter when there is a liquid filled in the structure but there is less change for the $\mathrm{L}(0,1)$ wave mode.

The factors to be considered for selecting a wave mode for $\mathrm{UGW}$ inspection as a probing mode are, dispersion, attenuation, excitability, and sensitivity [5], [11]. This study will investigate the potential of using $\mathrm{L}(0,1)$ for the inspection of pipelines. There are research performed to study and counteract for dispersion [4] and attenuation [8] of the $\mathrm{L}(0,1)$ wave mode. Based on this knowledge, it is be possible to obtain a sufficient test range with the $\mathrm{L}(0,1)$ wave mode for $\mathrm{UGW}$ inspection. With the current knowledge on the level of attenuation in $\mathrm{L}(0,1)$ and dispersion compensation techniques, the $\mathrm{L}(0,1)$ wave mode can be used as a probing mode for the UGW inspection. However, in the literature no attention has been given to study the potential of purity of excitation and flaw sensitivity of $\mathrm{L}(0,1)$.

In the present paper, properties and behaviour of the $\mathrm{L}(0,1)$ wave mode are examined and the advantages and disadvantages of its use in pipeline inspection are discussed with concentration on mode purity and flaw sensitivity. Investigation on pure excitation (mode purity) and high resolution (flaw sensitivity) of the $\mathrm{L}(0,1)$ wave mode is performed and compared with $\mathrm{L}(0,2)$ and $\mathrm{T}(0,1)$ numerically. The numerical results are validated empirically by aid of a 3D-Laser Doppler Vibrometry (3D-LDV) [12] experiments for quantitative measurements.

The paper is organized as follows: Section II, reviews the current UGW transducers. Then the Finite Element Analysis (FEA) on the excitation of the $\mathrm{L}(0,1)$ in isolation and the flaw sensitivity are documented in Section III. The experimental results and the discussion are reported in Section IV followed by the conclusions.

\section{Analysis of the CURREnt UGW TRAnSDUCERS}

The ability to effectively excite and isolate the desired wave modes evidently affects the data interpretation of the UGW inspection. The piezoelectric Thickness-Shear Transducers (TSTs) are the most commonly used commercial transducers for the UGW inspection. The TSTs are used to excite an in-plane vibration that will give rise to a propagating wave mode. If the TSTs are aligned axially, they excite the longitudinal guided wave modes (those possible for the operating frequency). For example, a $40 \mathrm{kHz}$ excitation toneburst on a nominal 8 inch schedule 40 steel pipe will generate $\mathrm{L}(0,1)$ and $\mathrm{L}(0,2)$. Whereas, if the TSTs are aligned circumferentially, torsional wave modes are excited (again those which are possible for the operating frequency). The excitation of two modes in the longitudinal transducer alignment complicates the inspection results, so conventionally another axially separated rings of transducers are used to suppress spurious wave mode (L $(0,1)$ wave mode) [13]. A 3D-LDV experiment has been performed to analyse the waveforms generated by the commercially available UGW TSTs. A laboratory experiment 


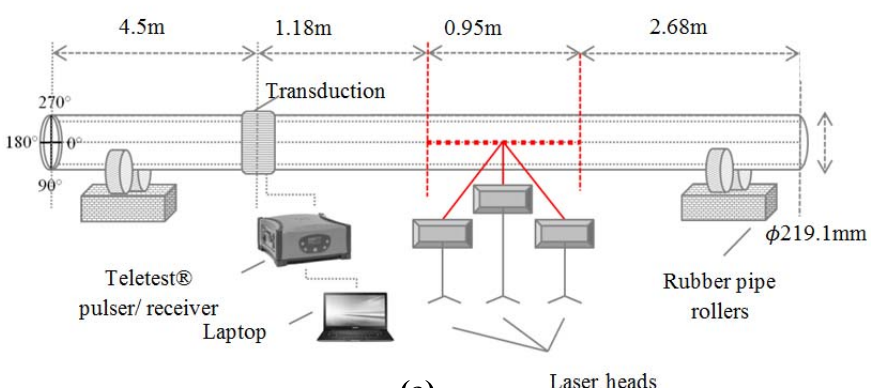

(a)

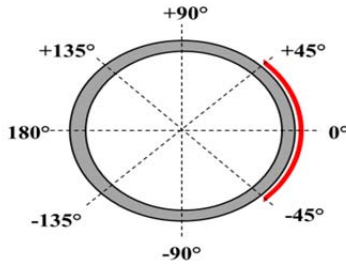

(b)

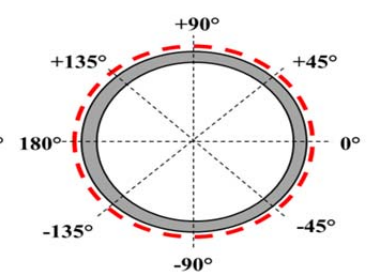

(c)
Fig. 2. (a) 3D-LDV experimental setup. (b) Schematics of the area scan. (c) Schematics of the line scan.

has been performed on a nominal 8 inch schedule 40 steel pipe (outer diameter: $219.1 \mathrm{~mm}$ and wall thickness: $8.18 \mathrm{~mm}$ ). The waveforms generated by a ring of [13] commercial UGW transducers (aligned both longitudinally and circumferentially) are monitored by $1.5 \mathrm{~m}$ away from the excitation. The experimental setup is illustrated in Fig. 2-a.

A Teletest@ Focus pulser/receiver [13] has used for excitation and a Polytec PSV-400-3D-M [12] scanning laser vibrometer was used to obtain 3D-LDV measurements of the vibration of the pipe surface. The 3D-LDV is equipped with three laser sensor heads in order to detect the 3D motions caused by the UGW propagation. The laser can detect the displacement and the surface velocity of propagating sound waves with a continuous analogue voltage output that is proportional to the target velocity component along the direction of the laser beam [14]. Hardware used is controlled by a PC so that the data acquisition is automatic. The pipe is resting on two pipe rollers and they are made out of rubber to minimize their influence on the waves propagating in the pipe.

Scanning length is calculated by the following formula in order to capture the full length of the waveform. Given the phase velocity for the $\mathrm{L}(0,1)$ mode, $V p h$, excitation frequency, $f$, and number of cycles of the excited wave, $n$, the pulse length, $L$, can be written as,

$$
L=n\left(\frac{V_{p h}}{f}\right)
$$

Therefore, the length of the line scan should be equal or greater than $L$.

In reference to cylindrical coordinate system, displacement of a wave propagating in the axial direction $z$ can be expressed as [9]:

$$
\begin{aligned}
& U_{r}=\cos (k z+\omega t) \cos (m \theta) \\
& U_{\theta}=\cos (k z+\omega t) \sin (m \theta) \\
& U_{z}=\sin (k z+\omega t) \cos (m \theta)
\end{aligned}
$$

where $U_{r}, U_{\theta}$ and $U_{z}$ are the radial, circumferential and axial displacements in reference to the cylindrical coordinate system respectively, $m$ is the circumferential order of the mode, $k$ is the wave number, $t$ is time, and $\omega$ is angular frequency.

To illustrate the difference between the two orientations of the transducers and behaviour of the waveforms generated, an area scan has been performed to cover $90^{\circ}$ of the pipe circumference (Fig. 2-b) along a length of $0.95 \mathrm{~m}$ of the structure. Ten cycles $40 \mathrm{kHz}$ Hann-windowed pulse has been applied using 24 evenly spaced transducers around the circumference. Fig. 3-a shows the measured waveforms as generated by circumferentially aligned TSTs and demonstrates the received wave mode has pure circumferential displacement (time of arrival of $\mathrm{T}(0,1))$. Two wave modes are observed in Fig. 3-b on the respective time of arrivals of $\mathrm{L}(0,2)$ and $\mathrm{L}(0,1)$ (as indicated by blue and red dashed vertical lines respectively). The $\mathrm{L}(0,2)$ wave mode has predominantly axial displacement (according to Fig. 3-b, it is displaced only in U3 direction) and the $\mathrm{L}(0,1)$ wave mode has both axial and radial displacements (according to Fig. 3-b, it is displaced in both U1 and U3 directions). Axially aligned TSTs generates in-plane vibration (Fig. 4-a) which leads to excite both $\mathrm{L}(0,2)$ and $\mathrm{L}(0,1)$ due to the axial particle displacement of both modes. At these frequencies $\mathrm{L}(0,2)$ has relatively low out-ofplane displacement. It is expected that an out-of-plane vibration would significantly bias the excitation of $\mathrm{L}(0,1)$. Therefore a transducer with a compression/out-of-plane vibration (Fig. 4-b) should preferentially excite $\mathrm{L}(0,1)$ in isolation. In section III-A, FEA has been conducted to investigate the potential of exciting the $\mathrm{L}(0,1)$ wave mode in isolation. The excitation of $\mathrm{L}(0,1)$ in isolation is vital to ease the data interpretation as $\mathrm{L}(0,2)$ is a faster (higher velocity) wave mode than $\mathrm{L}(0,1)$ (refer Fig. 1).

\section{Finite Element AnAlysis}

The FEA has been performed to study the potential of exciting $\mathrm{L}(0,1)$ in isolation and to achieve high defect sensitivity with focusing due to the presence of a larger number of flexural modes compared to $\mathrm{T}(0,1)$ and $\mathrm{L}(0,2)$. With increased complexity of geometries and analysis, the analytical calculations are no longer practical [15]. A 3D model was built using ABAQUS/EXPLICIT version 6.13 Finite Element (FE) software [16]. A hollow cylinder was modelled as a nominal 8 inch schedule 40 steel pipe (outer diameter: $219.1 \mathrm{~mm}$ and wall thickness: $8.18 \mathrm{~mm}$ ) with an axial length of $2.5 \mathrm{~m}$. The material properties used for steel were assumed as follows: Density $(\rho)=7830 \mathrm{~kg} / \mathrm{m} 3$, Young's modulus $(\mathrm{E})=207 \mathrm{GPa}$ and Poisson's ratio $(\mathrm{V})=0.3$. A linear eight node brick element (C3D8) has been used to reduce the computation time. Number of elements was calculated to use at least 8 elements to represent the smallest wavelength in the operating frequency. This type of mesh refinement has been adequately used in previous studies [17], [19]. Equally spaced, 24 circumferential points were selected for transmission and reception. To avoid reflections from the free edge of the pipe, an absorption region was used as shown in Fig. 5 without modelling a lengthier pipe for computational efficiency. 
(a)

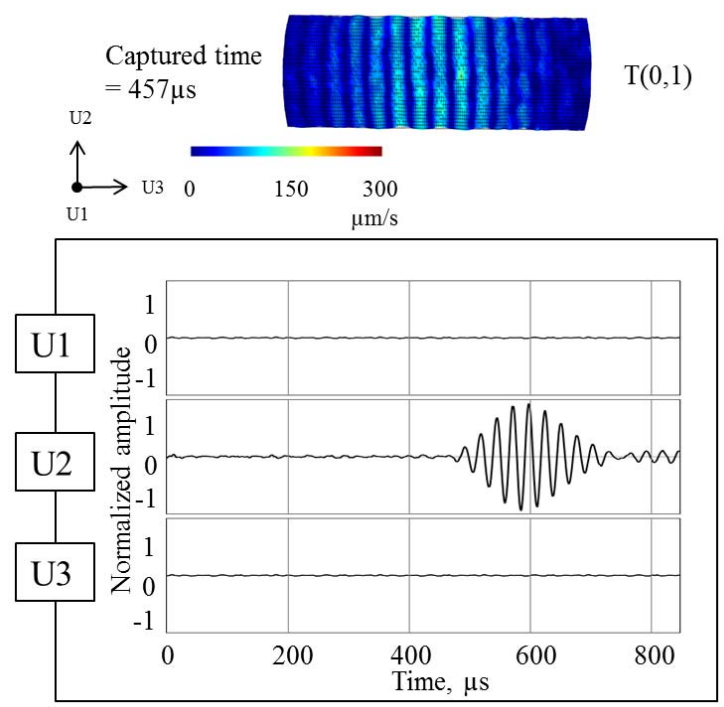

(b)
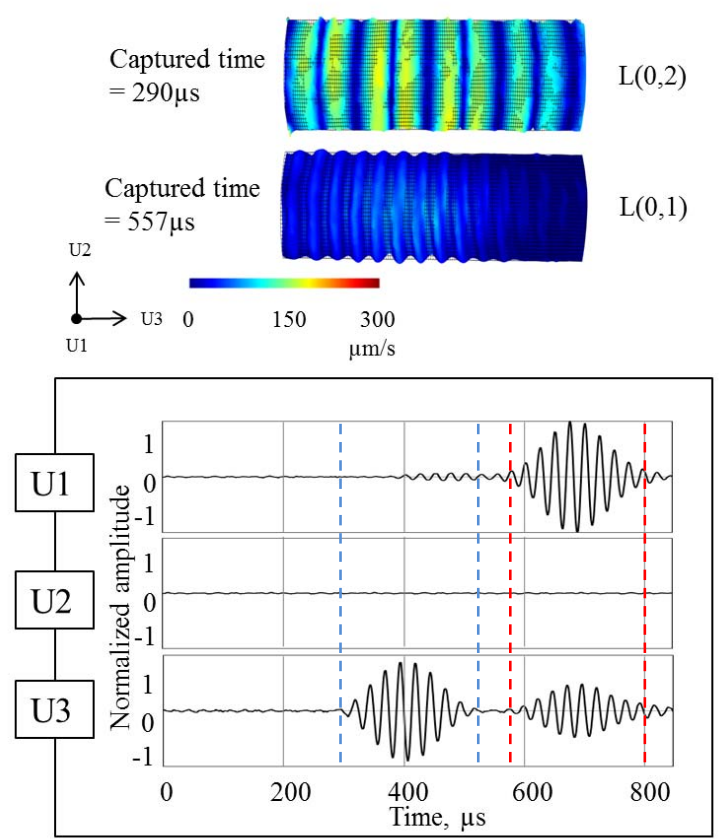

Fig. 3. 3D-LDV experimental results of waveforms generated by commercial UGW transducers aligned (a) circumferentially and (b) axially (normalized to the highest amplitude) U1, U2, and U3 represents radial, circumferential and axial displacement, respectively, caused by the applied vibration. Blue dashed lines represents $\mathrm{L}(0,2)$ wave mode and red dashed lines represents $\mathrm{L}(0,1)$ wave mode. These waveforms were monitored $1.5 \mathrm{~m}$ away from the transmission position.

(a)

\section{Undeformed}

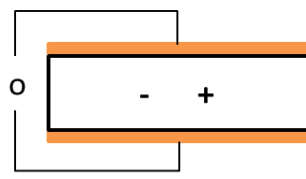

(b)

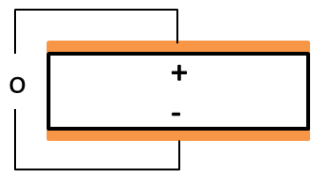

Deformed

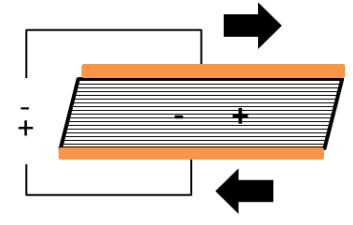

$\downarrow$

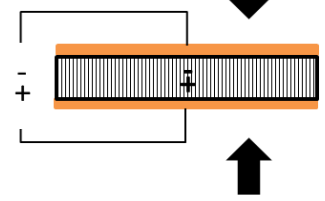

Fig. 4. Schematics of the vibration direction and electron arrangement of (a) thickness shear piezoelectric element and (b) compression piezoelectric element.

The absorbing boundary was achieved by use of the 'infinite element' ((ABAQUS element type CIN3D8) [16].) in Abaqus. This consists of elements defined over semi-infinite domains with suitably chosen decay functions [20]. The vibration of the incident pulse propagates continuously in to the absorption elements without any reflection.

\section{A. Excitation of the $L(0,1)$ in Isolation}

As explained in sections I \& II, the $\mathrm{T}(0,1)$ mode has circumferential displacement. Whereas, $\mathrm{L}(0,2)$ has predominantly axial displacement and $\mathrm{L}(0,1)$ has radial displacement and low axial displacement [2], [3]. Based on wave mode behaviour,

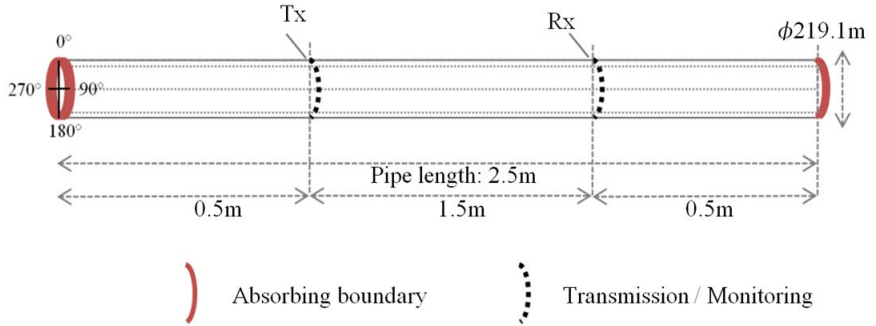

Fig. 5. Layout of the finite element model.

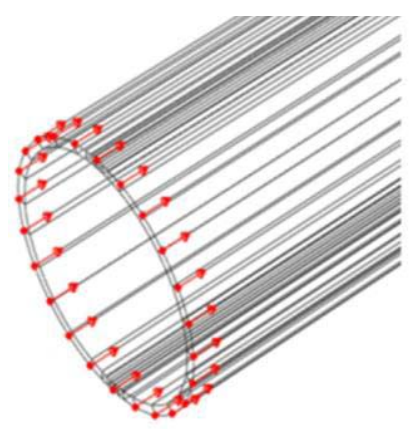

(a)

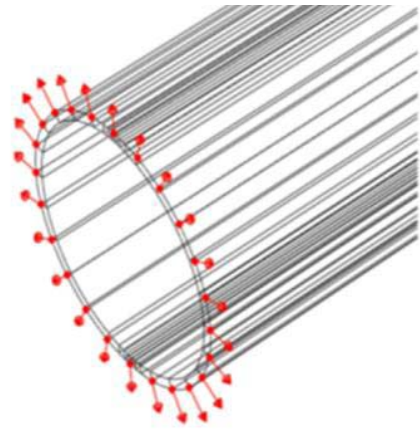

(b)
Fig. 6. Applied load (a) shear vibration and (b) compression vibration.

the $\mathrm{T}(0,1)$ wave mode can be excited in isolation by circumferentially aligned TSTs. Whereas, the $\mathrm{L}(0,2)$ wave mode can be generated by a longitudinally aligned TSTs. Due to the axial (in-plane) displacement of $\mathrm{L}(0,2)$ and $\mathrm{L}(0,1)$, commercial TSTs excite both wave modes which means an additional ring 

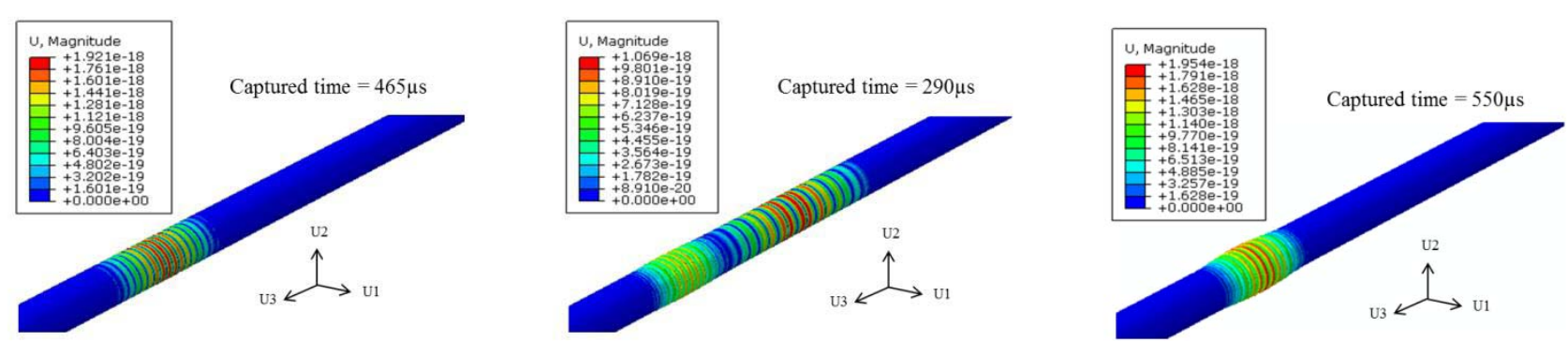

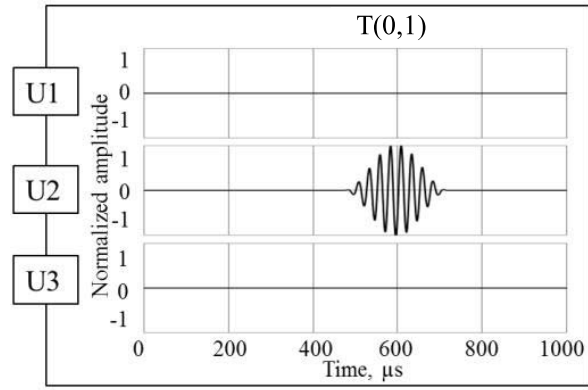

(a)

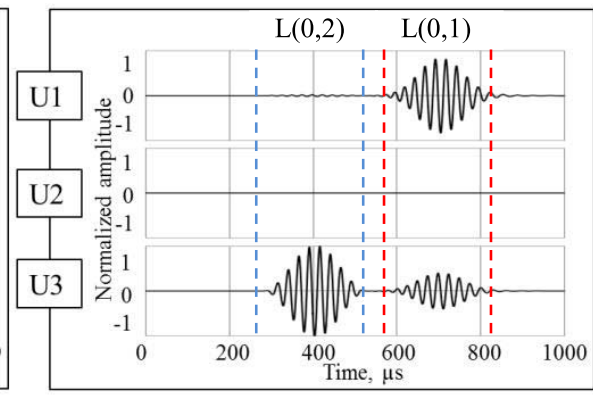

(b)

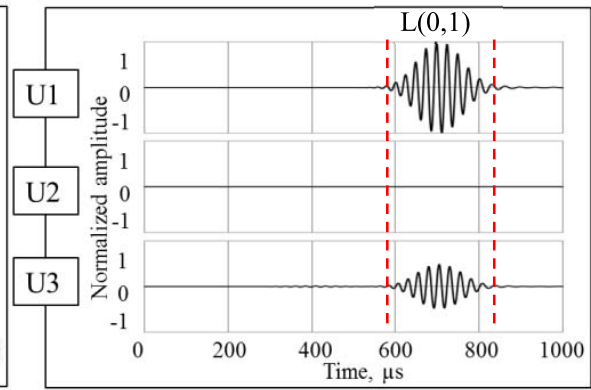

(c)

Fig. 7. Monitored waveforms generated by (a) circumferential vibration, (b) longitudinal vibration, and (c) compression vibration. U1, U2, and U3 represent radial, circumferential and axial displacement, respectively, caused by the applied vibration. Blue dashed lines represents $\mathrm{L}(0,2)$ and red dashed lines represents $\mathrm{L}(0,1)$. These waveforms were monitored $1.5 \mathrm{~m}$ away from the transmission position.

of transducers are required to suppress spurious wave mode. These additional rings of transducers increase the cost and weight of the commercial tooling.

Based on the experimental results in Section II, a FE model was performed to study the waveforms generated by current commercially available UGW transducers by applying in-plane shear vibration circumferentially and longitudinally as illustrated in Fig. 6-a. Then another FE model was performed to study the waveform generated by applying compression vibration (out-of-plane) as illustrated in Fig. 6-b. For this specific example, a $40 \mathrm{kHz}, 10$-cycle Hann-windowed pulse was excited at transmitting points $0.5 \mathrm{~m}$ away from the pipe edge. The generated waveforms in the FE models were monitored $1.5 \mathrm{~m}$ away from the transmission (Fig. 5). Excitation was applied using equally spaced 24 points around the circumference to suppress non-axisymmetric guided wave modes and transmit only the axisymmetric modes in the UGW frequency region.

Fig. 7 illustrates the predicted time domain data from the FE models and displacement caused by each transducer arrangement (in-plane circumferential, axial vibration and out-of-plane vibration, respectively). The FE results in Fig. 7-a and 7-b agrees with the displacement measured by the 3D-LDV in Section II (Fig. 3). Fig. 7-a illustrates the waveforms generated by circumferentially aligned shear vibration. Pure $\mathrm{T}(0,1)$ wave mode was excited due to the $\mathrm{T}(0,1)$ been the only axisymmetric wave mode with circumferential displacement in the UGW operating frequency region (20-100kHz). Fig. 7-b, illustrates the waveforms generated by longitudinally aligned TSTs. Both $\mathrm{L}(0,1)$ and $\mathrm{L}(0,2)$ were generated due to the axial displacement in both modes. Furthermore, it is evident from the Fig. 3-b and Fig. 7-b that the $\mathrm{L}(0,2)$ wave mode has predominantly axial displacement.

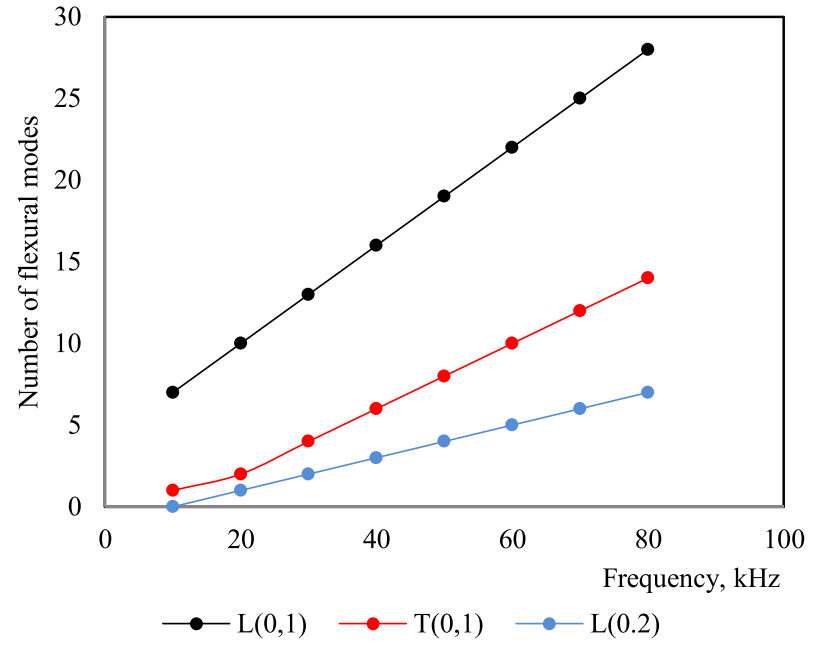

Fig. 8. Variation of the number of flexural wave modes with corresponding frequency of a nominal 8 inch schedule 40 (outer diameter: $219.1 \mathrm{~mm}$ and wall thickness: $8.18 \mathrm{~mm}$ ).

Therefore compression (out-of-plane) transduction suppresses the $\mathrm{L}(0,2)$ wave mode and transmit the $\mathrm{L}(0,1)$ wave mode in relative isolation (Fig. 7-c). Based on this there is no need for additional rings to suppress the $\mathrm{L}(0,2)$ wave mode and data interpretation will be easier due to the mode purity.

\section{B. Resolution of the L $(0,1)$ Wave Mode (Flaw Sensitivity)}

The main objectives of the structural inspections are to achieve high sensitivity for anomalies present in the structure under inspection. One way to enhance the sensitivity is through sound energy focusing at a predetermined region of the structure. The sound energy focusing is a method of tuning 


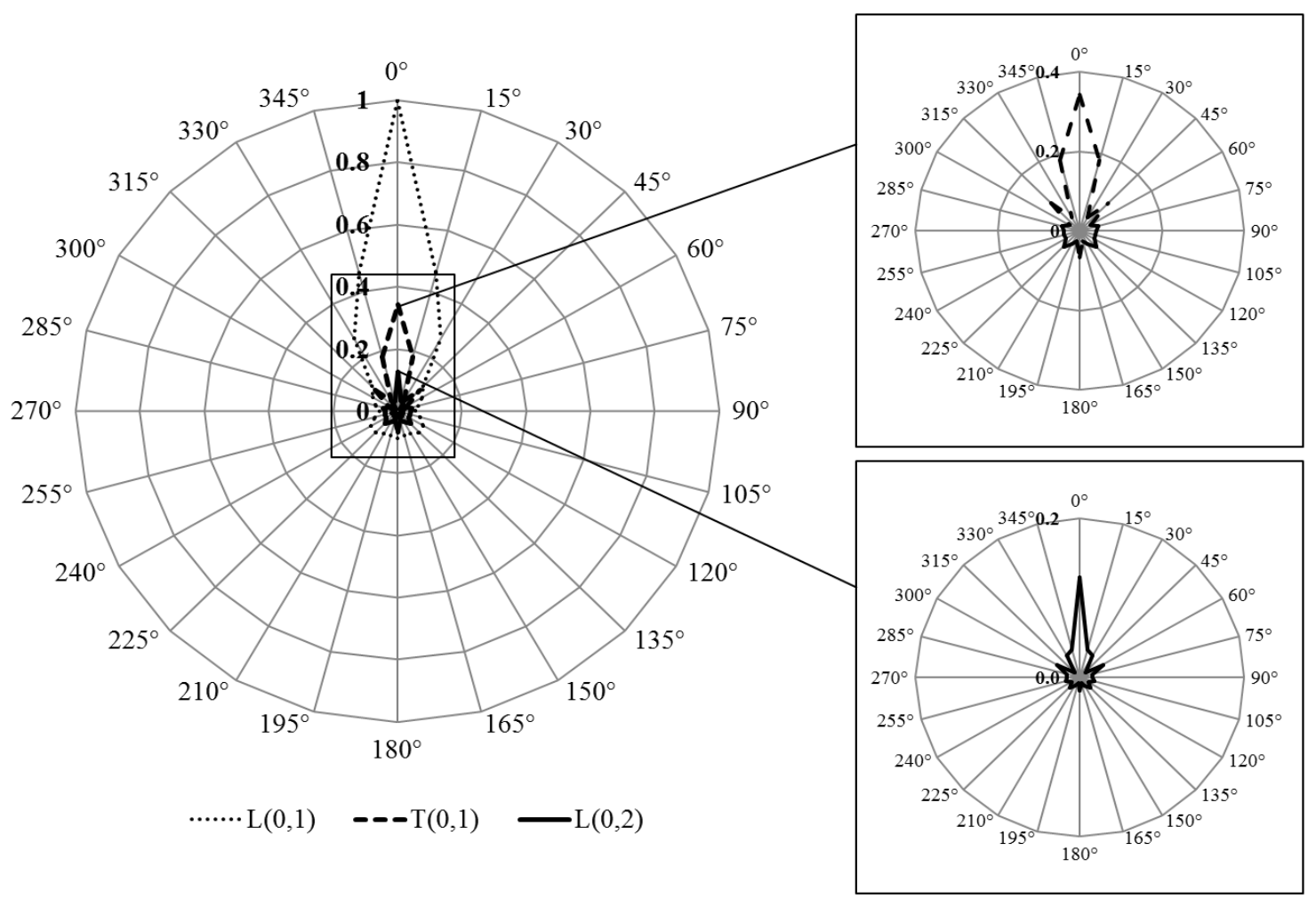

Fig. 9. Polar plots of the FE result of stresses achieved in AF technique on a nominal 8 inch schedule 40 (outer diameter: $219.1 \mathrm{~mm}$ and wall thickness: $8.18 \mathrm{~mm}$ ) steel pipe at the axial focal position (normalized to the maximum amplitude of all the 3 cases).

ultrasonic energy at a targeted point of the structure under inspection [21]-[26]. The technique works by superimposing an axisymmetric wave mode with a number of flexural wave modes to create a focal spot at the desired location. Focusing is achieved by timed transmission of each mode using time delays and scaling factors, such that the modes coalesce at one point in time at the focal point. The sound energy can also be focused as a post processing focusing technique (Synthetic Focusing) [27]. The performance of the energy focusing techniques depends on the number of flexural modes in the operating frequency [28]. Every axisymmetric wave mode $(X(0, m))$ has an associated family of flexural wave modes $(F(n, m))$ as appear in blue in Fig.1. For a given frequency $(20-100 \mathrm{kHz})$, the $\mathrm{L}(0,1)$ wave mode has a higher number of associated excitable flexural wave modes compared to $\mathrm{L}(0,2)$ and $\mathrm{T}(0,1)$. Therefore, the $\mathrm{L}(0,1)$ wave mode and its family of flexural wave modes has the potential to provide greater sensitivity for anomalies [28], [29].

Dispersion curves for a nominal 8 inch schedule 40 (Fig. 1) steel pipe has been used to illustrate the higher number of flexural wave modes associated with the $\mathrm{L}(0,1)$ mode. The number of flexural wave modes at a range of frequencies have been recorded for each of $\mathrm{L}(0,1), \mathrm{T}(0,1)$ and $\mathrm{L}(0,2)$ wave mode families. This is shown graphically in Fig. 8. In all considered frequencies (typical UGW operating frequencies), the $\mathrm{L}(0,1)$ wave mode has four times more flexural wave modes than the $\mathrm{L}(0,2)$ and two times more flexural wave modes than the $T(0,1)$ wave mode. The higher numbers of flexural wave modes are likely to result in better sensitivity when applying $\mathrm{L}(0,1)$ to a focusing technique [22]. This indicates that, there are potentially beneficial properties of the $\mathrm{L}(0,1)$ wave mode to increase the defect amplitude and achieve higher flaw sensitivity of UGW inspection.

Additionally, there has been research presented recently on the possibility of using UGW to determine the circumferential and through-wall extent of flaws [30]. The performance of these flaw sizing techniques are limited to the number of reflected flexural wave modes. Therefore, having a higher number of flexural wave modes in the $\mathrm{L}(0,1)$ family can extends the potential capability of the flaw sizing techniques.

An empirically validated Active Focusing (AF) technique [29], [31] has used to compare the focal point resolution of $\mathrm{L}(0,2), \mathrm{T}(0,1)$ and $\mathrm{L}(0,1)$ wave modes. The layout of the FE model is illustrated in Fig. 5. The focusing parameters were calculated for all the probing modes $(\mathrm{T}(0,1), \mathrm{L}(0,2)$ and $\mathrm{L}(0,1)$ ) by applying scaling factors and time delays for a 10 -cycle $40 \mathrm{kHz}$ Hann-windowed pulse [31].

The focusing parameters for the probing modes were transmitted at 24 equally spaced points individually using three different FE models and monitored at the focal point $1.5 \mathrm{~m}$ away from the transmission point. Applying time delays and scaling factors will excite all the modes in the respective family of flexural modes at the point of interest. All the modes will superposed and generate a focal spot. Fig. 9 illustrates the normalized FE results of the monitored focal spot for all the probing modes. The displacement amplitude of the focal 


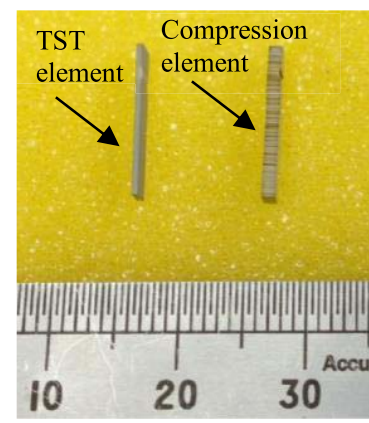

(a)

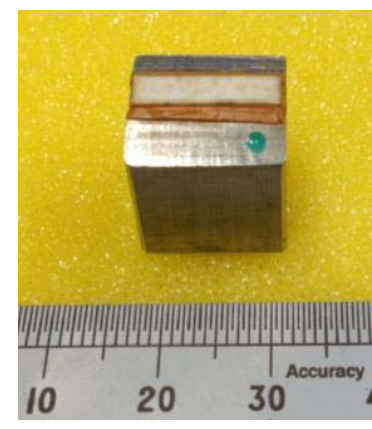

(b)
Fig. 10. (a) Commercial TST element and compression element. (b) Assembled compression transducer.

point around the circumference using the monitoring points (refer Fig. 5) are plotted (polar plot) for all the probing modes considered. According to FE results in Fig. 9, applying the $\mathrm{L}(0,1)$ wave mode for an energy focusing technique enhances the flaw sensitivity of UGW inspection by approximately 2.5 times compared to $\mathrm{T}(0,1)$ and by approximately 5 times compared to $\mathrm{L}(0,2)$. These results exhibit higher potentials of using the $\mathrm{L}(0,1)$ wave mode for inspection of pipelines.

\section{EXPERIMENTAL RESULTS AND DISCUSSION}

Laboratory experiments have been performed on a nominal 8 -inch schedule 40 steel pipe (outer diameter: $219.1 \mathrm{~mm}$ and wall thickness: $8.18 \mathrm{~mm}$ ) to validate the FE model results in Section III-A \& B. Same experimental setup illustrated in Section II (Fig. 2) has used for the experimental validation of exciting $\mathrm{L}(0,1)$ in isolation and demonstrates the high flaw sensitivity of $\mathrm{L}(0,1)$. Hann-windowed $40 \mathrm{kHz}$ pulse has been applied using circumferentially evenly spaced TSTs and compression transducers. Compression transducers were manufactured by Smart Material Corp [32] using "1-3 random fiber composite" piezo-ceramic elements for the dimension $(13 \mathrm{~mm} \times 3 \mathrm{~mm} \times 1 \mathrm{~mm}$, width, height and depth respectively) which can be fit in the backing block of the current commercial TSTs. So these transducers can be used with the existing UGW inspection tooling. The random fiber composites are chosen due to the reduced acoustic impedance, high coupling factor and the random element distribution yield increased spurious mode suppression due to the random spacing [32]. Furthermore, 3D-LDV has used to scan along a $0.95 \mathrm{~m}$ line on the pipe surface and pipe has rotated 24 times with $15^{\circ}$ intervals (Fig. 2-c) using pipe rollers to gain 24 individual line scans at different circumferential positions. Hann-windowed $40 \mathrm{kHz}$ AF focusing parameters $(\mathrm{L}(0,1)$ and $\mathrm{T}(0,1))$ has applied for excitation.

There is a good agreement with the predicted FE results in Section III and experimental results measured by the 3D-LDV displacement derived from different circumferential positions and area scans. The experimental validation of FEA in Section III-A are illustrated in Fig. 3 and 11. It represents the surface velocity of the waveguide at a fixed point in time caused by the excitation of $40 \mathrm{kHz}$ Hann-windowed pulse circumferentially evenly spaced 24 axially aligned TSTs (Fig. 3-b) and compression transducers (Fig. 11).
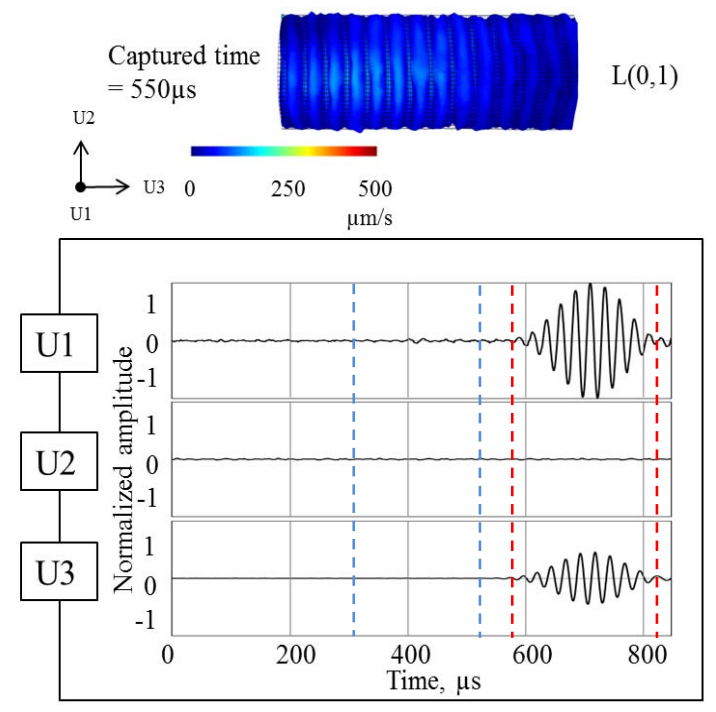

Fig. 11. 3D-LDV experimental results of waveform generated by compression transducers.

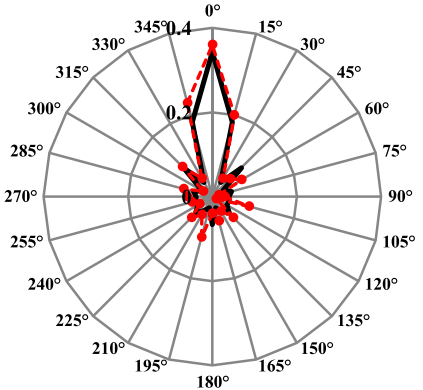

(a)

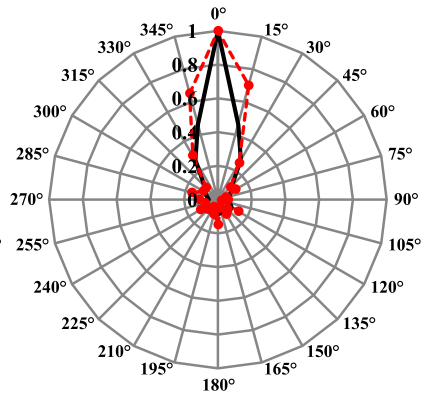

(b)
Experimental Results

Fig. 12. 3D-LDV experimental results of focal point monitored by applying $\mathrm{AF}$ technique for (a) $\mathrm{L}(0,1)$. and (b) $\mathrm{T}(0,1)$.

The measured particle displacement of the waveguide that were measured by the 3D-LDV correlates with the predicted displacement from the FE models in Section III-A with a good agreement. Based on these results, the most adequate way to excite the $\mathrm{L}(0,1)$ wave mode in isolation for pipe inspection is using transduction with compression vibration. The $\mathrm{L}(0,2)$ wave mode is suppressed due to the out-of-plane vibration (there is no axial particle displacement Fig. 4-b \& 11). Therefore, there is no requirement of additional rings of transducers to suppress the $\mathrm{L}(0,2)$ wave mode. This reduces the cost and weight of UGW inspection tools. Fig. 10 photographically illustrates the difference of commercial TST element and compression element.

Corresponding experimental validation of the FEA in Section III-B are illustrated in Fig. 12 which were measured by 3D-LDV line scan experiment. The transmitted AF focusing parameters were monitored at $1.5 \mathrm{~m}$ away from the transmission. The derived focal point information for $\mathrm{L}(0,1)$ and $\mathrm{T}(0,1)$ are compared in Fig. 12 . The $\mathrm{L}(0,2)$ wave mode has not been considered as it has low focal amplitude (Fig. 9) due to having fewer number of flexural wave modes. The 
amplitude of the AF focal point FEA results and corresponding experimental validation illustrates that the $\mathrm{L}(0,1)$ wave mode has approximately 2.5 times amplitude gain than the $\mathrm{T}(0,1)$ wave mode and approximately 5 times than the $\mathrm{L}(0,2)$ wave mode.

\section{CONCLUSION}

The aim of the work presented was to study the potential of adopting the $\mathrm{L}(0,1)$ wave mode for UGW inspection of pipelines. In the past, less attention has been given to the UGW inspection using the $\mathrm{L}(0,1)$ wave mode due to its dispersive and attenuative properties. However, dispersion could be counteracted by selecting an appropriate frequency and/or dispersion compensation techniques [4]. Attenuation is considerably low at $20-100 \mathrm{kHz}$ frequency regions so it is possible to create the test with low attenuation [8]. Therefore, a combination of careful frequency selection and application of appropriate signal processing procedures make inspection with the $\mathrm{L}(0,1)$ wave mode a viable prospect.

In the present paper it was demonstrated that, under the investigated conditions, application of the $\mathrm{L}(0,1)$ wave mode for UGW inspection can enhance the flaw sensitivity of approximately 2.5 times compared to the $\mathrm{T}(0,1)$ wave mode and approximately 5 times compared to the $\mathrm{L}(0,2)$ wave mode. This results exhibits higher potentials of using the $\mathrm{L}(0,1)$ wave mode for UGW inspection of pipelines. This enhances the capabilities of UGW flaw detection and sizing. Furthermore, suitable transduction technique has been proposed for exciting the $\mathrm{L}(0,1)$ wave mode in isolation. It was validated that the out-of-plane transducers are capable of exciting the $\mathrm{L}(0,1)$ wave mode in isolation. This eliminates the need of having additional rings of transducers to suppress $\mathrm{L}(0,2)$. This reduces the cost and weight of UGW inspection tooling.

\section{ACKNOWLEDGMENT}

The authors gratefully acknowledge TWI Ltd. and the Centre for Electronic System Research (CESR) of Brunel University for funding that made this study possible. They are also grateful to Prof. P. Mudge, Dr. Bhavin Engineer, Mr. Sina Fateri of Plant Integrity Ltd., and Mr. Ignacio Garcia de Carellan of Brunel Innovation Centre for their constructive inputs and useful discussions.

\section{REFERENCES}

[1] A. H. Meitzler, "Mode coupling occurring in the propagation of elastic pulses in wires," J. Acoust. Soc. Amer., vol. 33, no. 4, pp. 435-445, 1961.

[2] M. G. Silk and K. F. Bainton, "The propagation in metal tubing of ultrasonic wave modes equivalent to Lamb waves," Ultrasonics, vol. 17 , no. 1, pp. 11-19, 1979.

[3] J. L. Rose, "Standing on the shoulders of giants: An example of guided wave inspection," Mater. Eval., vol. 60, no. 1, pp. 53-59, 2002.

[4] P. D. Wilcox, "A rapid signal processing technique to remove the effect of dispersion from guided wave signals," IEEE Trans. Ultrason., Ferroelectr., Freq. Control, vol. 50, no. 4, pp. 419-427, Apr. 2003.

[5] P. D. Wilcox, M. J. S. Lowe, and P. Cawley, "Mode and transducer selection for long range Lamb wave inspection," J. Intell. Mater. Syst. Struct., vol. 12, no. 8, pp. 553-565, 2001.

[6] R. Sanderson, "A closed form solution method for rapid calculation of guided wave dispersion curves for pipes," Wave Motion, vol. 53, pp. 40-50, Mar. 2014.

[7] H. Kwun, K. A. Bartels, and C. Dynes, "Dispersion of longitudinal waves propagating in liquid-filled cylindrical shells," J. Acoust. Soc. Amer., vol. 105, no. 5, pp. 2601-2611, 1999.
[8] W.-B. Na and H.-S. Yoon, "Wave-attenuation estimation in fluid-filled steel pipes: The first longitudinal guided wave mode," Russian $J$. Nondestruct. Test., vol. 43, no. 8, pp. 549-554, 2007.

[9] D. C. Gazis, "Three-dimensional investigation of the propagation of waves in hollow circular cylinders. I. Analytical foundation," J. Acoust. Soc. Amer., vol. 31, no. 5, pp. 568-573, 1959.

[10] D. C. Gazis, "Three-dimensional investigation of the propagation of waves in hollow circular cylinders. II. Numerical results," J. Acoust. Soc. Amer, vol. 31, no. 5, pp. 573-578, 1959.

[11] D. W. Greve, H. Sohn, C. P. Yue, and I. J. Oppenheim, "An inductively coupled Lamb wave transducer," IEEE Sensors J., vol. 7, no. 2, pp. 295-301, Feb. 2007.

[12] Polytec. (Dec. 5, 2011). PSV-400 Scanning Vibrometer. [Online]. Available: http://www.polytec.com/fileadmin/user_uploads/Products/ Vibrometers/PSV-400/Documents/OM_DS_PSV-400_2011_05_E.pdf, accessed Apr. 27, 2015.

[13] P. J. Mudge, "Field application of the Teletest long-range ultrasonic testing technique," Insight-J. Brit. Inst. Non-Destruct. Test., vol. 43 , no. 2, pp. 74-77, 2001.

[14] D. E. Oliver, "Tutorial: 3D scanning vibrometry for structural dynamics measurements," presented at the IMAC-XXVII, Conf. Expo. Struct. Dyn., Orlando, Feb. 9-12, 2011.

[15] A. J. Allen, M. T. Hutchings, C. M. Sayers, D. R. Allen, and R. L. Smith, "Use of neutron diffraction texture measurements to establish a model for calculation of ultrasonic velocities in highly oriented austenitic weld material," J. Appl. Phys., vol. 54, no. 2, pp. 555-560, 1983.

[16] Abaqus Version 6.13 User's Manual, Abaqus-SIMULIA, Waltham, MA, USA, 2013.

[17] D. N. Alleyne, M. J. S. Lowe, and P. Cawley, "The reflection of guided waves from circumferential notches in pipes," J. Appl. Mech., vol. 65, no. 3, pp. 635-641, 1998

[18] P. S. Lowe, S. Fateri, R. Sanderson, and N. V. Boulgouris, "Finite element modelling of the interaction of ultrasonic guided waves with coupled piezoelectric transducers," Insight-Non-Destruct. Test. Condition Monitor, vol. 56, no. 9, pp. 505-509, 2014.

[19] S. Fateri, P. S. Lowe, B. Engineer, and N. V. Boulgouris, "Investigation of ultrasonic guided waves interacting with piezoelectric transducers," IEEE Sensors J., vol. 15, no. 8, pp. 4319-4328, Aug. 2015.

[20] J. Lysmer and R. L. Kuhlemeyer, "Finite dynamic model for infinite media," J. Eng. Mech. Division, vol. 95, no. 4, pp. 859-878, 1969.

[21] J. L. Rose and P. J. Mudge, "Flexural mode focusing in pipe," in Proc. 8th Eur. Conf. Non-Destruct. Test, Barcelona, Spain, 2002, pp. 17-21.

[22] T. Hayashi, K. Kawashima, Z. Sun, and J. L. Rose, "Analysis of flexural mode focusing by a semianalytical finite element method," J. Acoust Soc. Amer., vol. 113, no. 3, pp. 1241-1248, 2002.

[23] C. Ennaceur, P. Mudge, B. Bridge, M. Kayous, and T. H. Gan, "Application of the time reversal technique to the focusing of long-range ultrasound in pipelines," Insight-Non-Destruct. Test. Condition Monitor., vol. 49 , no. 4 , pp. $217-223,2007$

[24] J. O. Davies, "Inspection of pipes using low frequency focused guided waves," Ph.D. dissertation, Imperial College, London, U.K., 2008.

[25] J. Li and J. L. Rose, "Natural beam focusing of non-axisymmetric guided waves in large-diameter pipes," Ultrasonics, vol. 44, no. 1, pp. 35-45, 2006.

[26] P. S. Lowe, R. Sanderson, N. V. Boulgouris, and T.-H. Gan, "Hybrid active focusing with adaptive dispersion for higher defect sensitivity in guided wave inspection of cylindrical structures," Non-Destruct. Test. Eval., 2015. [Online]. Available: http://dx.doi.org/10.1080/10589759. 2015.1093628

[27] J. Davies and P. Cawley, "Synthetic focusing for high resolution guided wave pipe inspection: Further results and robustness studies," in Proc. AIP Conf., Rev. Prog. Quant. Nondestruct. Eval., vol. 975. 2008, pp. 139-146.

[28] P. Catton, "Long range ultrasonic guided waves for the quantitative inspection of pipelines," M.S. thesis, School Eng. Design, Brunel Univ., Uxbridge, U.K., 2009.

[29] J. Mu, L. Zhang, J. L. Rose, and J. Spanner, "Defect sizing in pipe using an ultrasonic guided wave focusing technique," in Proc. AIP Conf., Rev. Prog. Quant. Nondestruct. Eval., vol. 820. 2006, pp. 760-766.

[30] R. M. Sanderson and P. P. Catton, "Flaw sizing in pipes using long-range guided wave testing," in Proc. QNDE Conf., San Diego, CA, USA, 2010, p. 689.

[31] J. Li and J. L. Rose, "Angular-profile tuning of guided waves in hollow cylinders using a circumferential phased array," IEEE Trans. Ultrason., Ferroelectr., Freq. Control, vol. 49, no. 12, pp. 1720-1729, Dec. 2002.

[32] Smart Material Corporation. (2015). 1-3 Random Fiber Composites. [Online]. Available: http://www.smart-material.com/13Crand-productmain.html, accessed Jan. 28, 2015. 


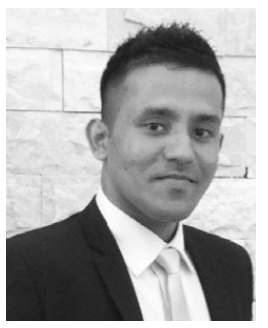

Premesh Shehan Lowe was born in Colombo, Sri Lanka. He received the B.Sc. (Hons.) in computer science and software engineering from Edith Cowan University, Perth, Australia, in 2008, and the M.Sc. degree in distributed systems and network engineering from the University of Hertfordshire, Hatfield, U.K., in 2012. He is currently pursuing the Ph.D. degree in electrical and computer engineering with Brunel University, London, U.K. In 2012, he joined the Integrity Management Group, TWI Ltd., Cambridge, U.K., as a Ph.D. Research Engineer. His research is focused on finite-element methods and signal processing of ultrasonic guided waves (UGW). His research interests are to develop novel UGW sensors to achieve higher flaw sensitivity, UGW energy focusing, flaw sizing in cylindrical structures, and UGW mode conversion from discontinuities.

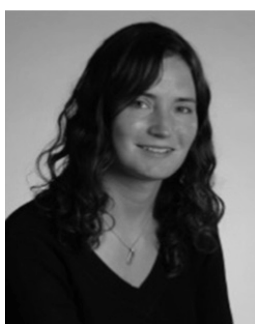

Ruth M. Sanderson was born in Cambridge, U.K. She received the B.Sc. (Hons.) degree in mathematics and the Ph.D. degree in mechanical engineering from the University of Warwick, in 1998 and 2012, respectively. Since 1998, she has been with TWI Ltd., Cambridge, as a Project Leader, where she is part of the Numerical Modeling and Optimization Section. During her time at TWI, she has gained experience in several areas of finite-element modeling, including general stress analysis and fracture mechanics. In addition to this, she has developed expertise in two key structural integrity areas, namely, weld modeling and simulation of ultrasonic wave propagation with an emphasis on long-range guided waves for nondestructive testing of pipelines.

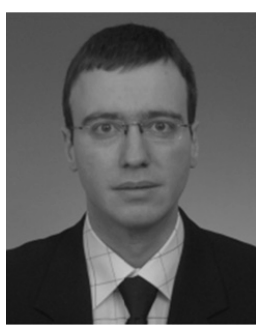

Nikolaos V. Boulgouris (S'96-M'04-SM'09) received the Ph.D. degree from the Electrical and Computer Engineering Department, University of Thessaloniki, Greece, in 2002. From 2003 to 2004, he was a Post-Doctoral Fellow with the Department of Electrical and Computer Engineering, University of Toronto, Canada. From 2004 to 2010, he served as a Lecturer and a Senior Lecturer with King's College London, U.K. He is currently a Senior Lecturer with the Department of Electronic and Computer Engineering, Brunel University, London, U.K. He is an Elected Member of the IEEE Image, Video, and Multidimensional Signal Processing Technical Committee. He served as a Guest Co-Editor of two journal special issues and was the Co-Editor of the book Biometrics: Theory, Methods, and Applications (Wiley-IEEE Press) in 2009. He is an Associate Editor of the IEEE TRANSACTIONS ON IMAge Processing. He served as an Associate Editor of the IEEE SignaL PROCESSING LETTERS from 2007 to 2010.

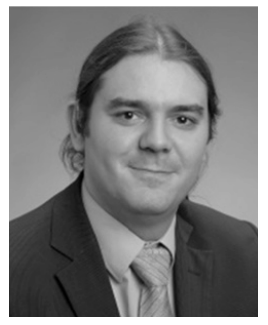

Alex G. Haig was born in London, U.K. He received the B.Sc. (Hons.) degree in computer science from the University of Essex, and the M.Sc. degree in advance computer science from the University of East Anglia, in 2004 and 2005, respectively. He conducted the Eng.Doc. in ultrasonic guided waves with Brunel University from 2006 to 2010. Since 2010, he has been with TWI Ltd., Cambridge, U.K., as a Project Leader where he is part of the Integrity Management Group. He has experience in research and development for long-range ultrasonic techniques and systems. During this time, his primary interests have been in analysis of ultrasonics, transducer selection, array design, signal processing, and software.

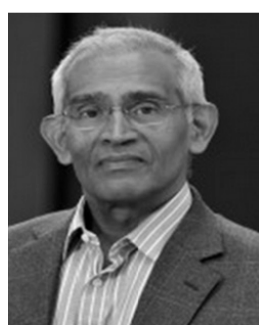

Wamadeva Balachandran (F'04) was a Visiting Professor with Driftmier Engineering Centre, University of Georgia, in 1993 and 1996, respectively. He has been a Visiting Professor with the University of Mansoura, Egypt, and Dongguan University, China, since 1993 and 1996, respectively. He served as the Head of the Department of Systems Engineering with Brunel University from 1999 to 2004. In 2004, he was a Visiting Scholar with the School of Engineering and Applied Science, University of California, Los Angeles. $\mathrm{He}$ is currently a Research Professor and the Director of the Centre for Electronic Systems Research, Brunel University, U.K. His research interest spans several different disciplines: electrostatics and charged particle dynamics, electrohydrodynamics, micro/nano technologies, microengineered devices for POCT, biosensors, fingerprint biometrics, global positioning satellite system for blind navigation, ultrasonic and EMAT NDT, electron beam and microwave plasma for environmental pollution control, and medical electronics. He is a Fellow of the Institution of Engineering and Technology (U.K.), the Institute of Physics (U.K.), the Institute of Measurement and Control (U.K.), and the Royal Society of Arts (U.K.). He has actively pursued research in these interdisciplinary fields for more than 30 years, publishing more than 350 papers to date, and filing 15 patent applications. He has presented more than 45 plenary and invited talks in his field of expertise at international conferences around the globe. He was a recipient of the IEEE John Melcher Best Paper Award and Innovation and Creativity Awards in 2000 and 2004. He continues to review manuscripts for 15 archival journals and research grant applications for EPSRC (U.K.), EU Framework, NSF (USA), NSERC (Canada), the Greek Government, Lockheed Martin, the Swiss Research Council, the Portuguese Research Council, and the Singapore Government. 